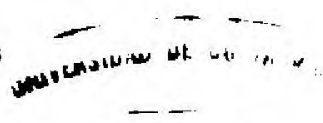

\title{
Leucine and Tissue Distribution of Butky and Small Neutral Amino Acids in Rats: Dissociation between Transport and Insulin- mediated Effects
}

\author{
C. de Céspedes*, J. G. Thoene, K. Lowler and H. N. Christensen \\ Departments of Biological Chemistry and Pediatrics, University of Michigan Medical \\ School, Ann Arbor, Michigan 48109, USA
}

\begin{abstract}
Summary: The mechanism of the observed decrease in the plasma concentration of several amino acids in the presence of high levels of Leu has remained unexplained. In the present study a decrease in the plasma concentration of lle, Val, Phe, Tyr, Met, Ala, Pro and Gly was observed after the intraperitoneal injection of Leu to weanling rats. Decreases in net intracellular concentrations in muscle accompanied the decrease in plasma of all of these amino acids except Pro and Gly. An increase in the distribution ratio muscle/plasma was observed exclusively for Gly after administration of Leu or of a non-insulinogenic transport system $\mathrm{L}$ analogue. Diazoxide suppressed the Leu-induced decreases in plasma and muscle intracellular concentrations of Ile and Val as well as of Pro in plasma. An increase in the distribution ratio liver/plasma was observed for Pro and Gly in the absence but not in the presence of diazoxide. All the above changes were statistically significant. Hence insulin probably mediates Leu effects, promoting an increased utilization of Ile and Val in muscle and of Pro in liver. A more direct effect of Leu appears to be involved in the apparent increased utilization of Phe, Tyr and Ala in the same tissue. Gly depletion in plasma can be explained by its trapping by inhibitory action of Leu on the exodus of Gly through transport system $L$.
\end{abstract}

High levels of Leu are usually accompanied by a decrease in the plasma concentrations of several amino acids. In maple syrup urine disease (McKusick 24860) (MSUD) Ile and $\mathrm{Val}$ are often decreased in plasma in the presence of high Leu levels, in spite of a common metabolic block affecting the catabolism of the three branched-chain amino acids (BCAA) (DiGeorge et al, 1982; Snyderman et al., 1964). Experimental administration of Leu results also in a decrease of Ile and Val, but other, mainly the bulky neutral amino acids also known as large neutral amino acids (LNAA), are also

*Correspondence: Institute of Research in Health, (INISA), University of Costa Rica, San José, Costa Rica, Central America

MS received 20.6.91 Accepted 9.10.91 
depleted in plasma. This effect has been observed in rats fed high-Leu diets (Rogers et al., 1962), after oral loads of Leu to children, either normal or with MSUD (Nyhan et al., 1961; Snyderman et al., 1984), as well as after intravenous Leu infusion to healthy adults (Eriksson et al., 1981) or to patients with hepatic encephalopathy (Rossi-Fanelli et al., 1986). In the latter, the Leu-induced decreases in the plasma concentrations of Phe and Tyr apparently result in clinical improvement (RossiFanelli et al., 1986).

The mechanisms of these decreases have, however, not been explained. Loss in the urine has been excluded as a cause (Nyhan et al., 1961). Indeed, it is likely that different mechanisms may apply to different amino acids or groups of amino acids. Leu is a well-known insulin secretagogue both in humans (Fajans et al., 1967) and rats (Landgraf et al., 1974), and insulin has long been known to cause a decrease in the concentrations of various amino acids in plasma (Luck et al., 1928), the BCAA being the most sensitive (Fukagawa et al., 1986). Transport effects have been proposed but not demonstrated (Nyhan et al., 1961; Eriksson et al., 1981). Eriksson et al. (1981) have suggested that exchange diffusion, a distinctive property of transport system $\mathbf{L}$ (Oxender and Christensen, 1963; Winter and Christensen, 1964), between high intracelfular Leu and plasma LNAA may be involved. Previous work in this laboratory has shown, furthermore, that system $\mathrm{L}$ in muscle (Christensen et al., 1948) and liver (Christensen and Cullen, 1981) apparently serves extensively for exodus of several neutral amino acids, previously concentrated into the cell by $\mathrm{Na}^{+}$-dependent transport systems (Oxender and Christensen, 1963) that operate mainly inwards in these tissues. One of us (H.N.C.) has proposed that this effect may explain the ability of Leu to decrease the neutral amino acids in plasma (Christensen, 1987). In fact, we have shown recently that this inhibitory mechanism applies for various small neutral amino acids, explaining for this group a similar decreasing effect of the Phe on their plasma levels (de Céspedes et al., 1989).

The aim of the present study was to test this hypothesis as well as to assess the role of insulin in the aforementioned effect of Leu on other plasma amino acids.

\section{EXPERIMENTAL PROCEDURES}

Materials: L-Leucine was obtained from Sigma Chemical $\mathrm{Co}$. The racemic aminoexo isomer of the 2-aminonorbornane-2-carboxylic acid, aminoexo-2-aminobicyclo$(2,2,1)$ heptane-2-carboxylic acid, often designated as a \pm$) \mathbf{B C H}$, but abbreviated here as $\mathbf{a B C H}$, was our own preparation (Tager and Christensen, 1972). A commercial preparation of diazoxide (Hyperstat IV, Schering Corp.) was used. All other reagents were of the best grade commercially available.

Animals: Twenty two-day-old Sprague-Dawley male weanling rats weighing 38$66 \mathrm{~g}$ were used for all the experiments. Rats were fasted for $20-24 \mathrm{~h}$ before each experiment.

Procedure: Leu or aBCH was injected intraperitoneally as an isotonic solution at a dose of $10 \mathrm{mmol} / \mathrm{kg}$ body weight. Control rats received an intraperitoneal injection 
of $0.85 \% \mathrm{NaCl}(\mathrm{w} / \mathrm{v})$ in the corresponding volumen. Diazoxide was also injected intraperitoneally, at a dose of $200 \mathrm{mg} / \mathrm{kg}$ body weight $15 \mathrm{~min}$ before Leu; control rats received an intraperitoneal injection of distilled water in the corresponding volumen (in no case higher than $0.7 \mathrm{ml}$ ).

Two hours after the Leu or aBCH injection the rats were anaesthetized with ether. Blood was taken from the exposed heart and collected in a heparinized tube. Plasma was separated and deproteinized with an equal volumen of $12 \%$ sulphosalicylic acid. Approximately $1 \mathrm{~g}$ of liver and $0.5 \mathrm{~g}$ of muscle from the left thigh were weighed and ground in a mortar containing sand and a $10 \%$ solution of sulphosalicylic acid at a volume 4 times the fresh weight of the tissue in grams. The homogenate was filtered through Whatman No. 1 paper. The protein-free filtrate was stored at $-20^{\circ} \mathrm{C}$ until analysis.

Analysis: Amino acids were measured with a model $119 \mathrm{CL}$ Beckman automatic amino acid analyser using a high-sensitivity standard solution from Sigma. Insulin was measured by radioimmunoanalysis, using a species-specific rat antibody, and glucose spectrophotometrically in the Ligand and the Biochemistry Core Laboratories, respectively, of the Diabetes Center of the University of Michigan Medical Center, Ann Arbor.

Calculations: For calculations of apparent concentrations of amino acids in cell water, the liver and muscle samples were taken to contain, respectively, $49 \%$ and $62 \%$ intracellular and $22 \%$ and $15 \%$ extracellular water (Christensen and Cullen, 1981; Lowry and Hastings, 1942). The distribution ratio tissue/plasma was the ratio $\mu \mathrm{mol} / \mathrm{kg}$ cell water to $\mu \mathrm{mol} / \mathrm{kg}$ extracellular water (Lowry and Hastings, 1942).

Statistical analysis: Differences in plasma levels, intracellular concentrations and distribution ratios between rats treated with Leu, Leu and diazoxide, or $\mathrm{aBCH}$, and control rats were calculated by the $t$-test using separate variances when the number of controls and treated animals differed (Wilkinson, 1986).

\section{RESULTS}

Plasma levels, net intracellular concentrations and distribution ratios of the amino acids studied after Leu administration, in the absence and in the presence of diazoxide are shown in Table 1 . Measurements were made in all cases at $2 \mathrm{~h}$ after injection because Ile, an amino acid whose distribution is well known to be particularly sensitive to Leu, was found in preliminary experiments to be strongly decreased in plasma by that time (data not shown).

Plasma levels as well as net intracellular concentrations in both liver and muscle of Leu itself showed statistically significant increases after Leu injection. These increments in Leu levels were about 3-5 times higher in the presence of diazoxide. The distribution ratio muscle/plasma for Leu showed a statistically significant decrease in the absence of diazoxide; otherwise the distribution ratios for Leu appeared unaffected. 
Table 1 Changes in relative concentrations and distribution of each of nine neutral amino acids produced after injecting Leu or Leu plus diazoxide into weaning rats

\begin{tabular}{lccccccc}
\hline & \multicolumn{3}{c}{ Leu } & & \multicolumn{3}{c}{ Leu + diazoxide } \\
\cline { 2 - 5 } \cline { 6 - 7 } \cline { 6 - 7 } & Plasma & Liver & Muscle & & Plasma & Liver & Muscle \\
\hline Leu & $473^{* *}$ & $337^{* *}$ & $270^{* *}$ & & $1525^{*}$ & $1621^{*}$ & $1467^{*}$ \\
$\quad$ Distribution ratio & & 0.77 & $0.58^{* * *}$ & & & 1.16 & 0.97 \\
Ile & $36^{*}$ & 101 & $34^{*}$ & & 90 & 171 & 82 \\
$\quad$ Distribution ratio & & $2.68^{* * *}$ & 0.96 & & 1.74 & 0.93 \\
Val & $34^{*}$ & 118 & $34^{* *}$ & & 59 & 90 & 51 \\
$\quad$ Distribution ratio & & $3.99^{* * *}$ & 1.15 & & & $1.67^{* * *}$ & 0.76 \\
Phe & $49^{* *}$ & 132 & $56^{* *}$ & & $40^{* *}$ & 101 & 43 \\
$\quad$ Distribution ratio & & $2.83^{* * *}$ & 1.39 & & & $2.64^{* * *}$ & 1.17 \\
Tyr & $43^{*}$ & 119 & $47^{* *}$ & & $45^{*}$ & 65 & $40^{*}$ \\
$\quad$ Distribution ratio & & $2.76^{* *}$ & 1.19 & & & 1.75 & 1.06 \\
Met & $40^{*}$ & 132 & 70 & & $47^{* *}$ & - & - \\
$\quad$ Distribution ratio & & $3.57^{* *}$ & 1.86 & & & - & - \\
Ala & $51^{*}$ & 72 & $60^{* *}$ & & $66^{* * *}$ & 63 & $70^{* * *}$ \\
$\quad$ Distribution ratio & & 1.59 & 1.29 & & & 0.96 & 1.18 \\
Pro & $48^{*}$ & 118 & 70 & & 67 & 157 & 73 \\
$\quad$ Distribution ratio & & $2.51^{* * *}$ & 1.69 & & 2.14 & 1.07 \\
Gly & $56^{* *}$ & 84 & 89 & & $59^{* *}$ & 90 & 97 \\
$\quad$ Distribution ratio & & $1.53^{* * *}$ & $1.61^{* * *}$ & & 1.29 & $1.53^{* *}$
\end{tabular}

The three values in each column show for plasma, liver, and muscle, respectively, the percentage of the control value attained in $2 \mathrm{~h}$ for each of these tissues for the amino acid named in column 1 . The number below the percentage value for each amino acid shows the calculated relative distribution ratio between the concentrations in the cellular and extracellular water, as described under Methods, for the indicated solid tissue. The number of animals varies from 6 to 7 , both for control and experimental groups. Asterisks indicate the statistical significance of changes: ${ }^{*} p<0.001,{ }^{* *} p<0.01,{ }^{* * *} p<0.05$

Without exception every other amino acid studied showed a statistically significant decrease in its plasma concentration after Leu injection. Only in the case of the other two branched-chain amino acids Ile and Val as well as of Pro was the decrease in plasma concentration prevented by diazoxide. lle and Val showed as well a statistically significant decrease in their net intracellular concentration in muscle that was prevented also by the previous injection of diazoxide. Distribution ratios of Ile and $\mathrm{Val}$ were perturbed in the liver, but not in muscle; the observed statistically significant increase in liver was not prevented by diazoxide.

The aromatic amino acids Phe and Tyr showed also a statistically significant decrease in their net intracellular concentrations in muscle, not prevented by diazoxide. Distribution ratios for Phe, Tyr and Met were also increased in liver but prevented by diazoxide only in the case of Tyr. Tissue Met co-eluted with Cys in the experiments with diazoxide and hence could not be measured.

Ala showed a statistically significant decrease in its net intracellular concentration in muscle, an effect insensitive to diazoxide. Pro and Gly showed a statistically significant increase in their respective distribution ratios for liver that was preventd by diazoxide. Gly and Pro were, among all other amino acids studied (Leu of course excluded from consideration), the only ones that did not show a statistically significant 
decrease in their net intracellular concentration in muscle. Gly, on the other hand, was the only amino acid that showed a statistically significant increase in its distribution ratio in muscle after Leu administration. This effect was insensitive to diazoxide.

Experiments parallel to those with Leu were made with the system $\mathrm{L}$ analogue $\mathrm{aBCH}$ (Figure 1). This isomer of $\mathrm{BCH}$ was selected because it is not an insulin secretagogue (Tager and Christensen, 1971) and hence could serve to dissociate that action from the suspected direct effects on amino acid transport (Oxender and Christensen, 1963; Christensen, 1987). Gly was the only amino acid that showed a statistically significant decrease in plasma concentration after aBCH. The only statistically significant change found in liver after $\mathrm{aBCH}$ was a decrease in the net intracellular concentration of Gly (data not shown). On the other hand, aBCH caused in muscle a statistically significant increase in the distribution ratios of Ala and Gly, reproducing the same distinctive effect of Leu for the latter (Figure 1).

The effect of Leu in the absence and in the presence of diazoxide on plasma glucose and insulin concentrations at different times is shown in Figure 2. As expected, Leu caused a decrease in the plasma concentration of glucose that was prevented by diazoxide (Figure 2A). The insulin/glucose ratio was increased by $345 \%$ of the control at $60 \mathrm{~min}$ after Leu injection; this effect was also largely prevented by diazoxide (Figure 2B).

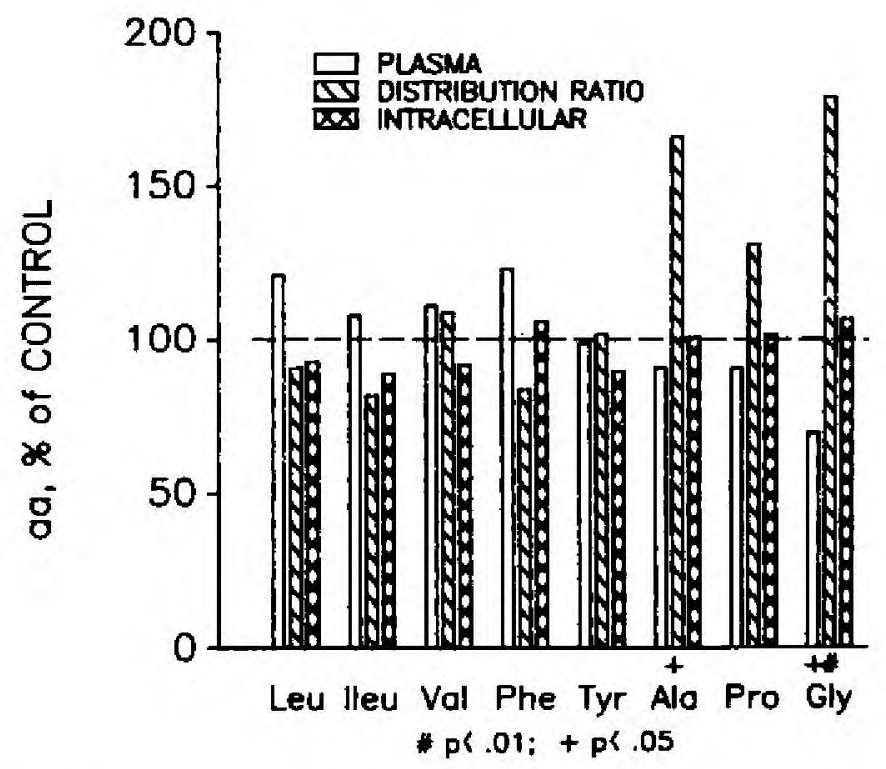

Figure 1 Plasma levels, distribution ratios, tissue/plasma, and net intracellular concentrations for muscle of neutral amino acids after aBCH injection. Data are shown as percentage of controls. $N$ varied from 7 to 13 animals for both controls and experimental groups 
A
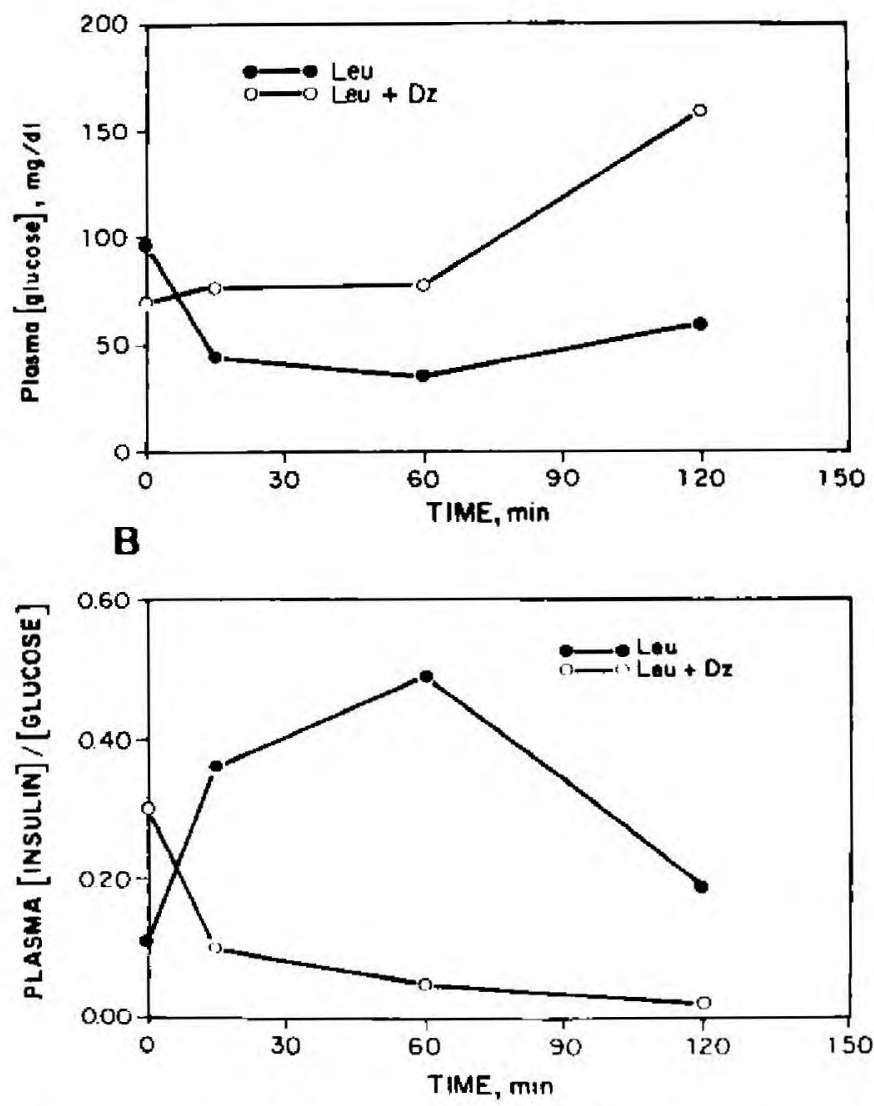

Figure 2 Time course of glucose concentration (A) and of insulin/glucose ratio (B) in plasma after Leu injection in the absence or in the presence of diazoxide $(\mathrm{Dz})$. Each point represents the average for three animals

\section{DISCUSSION}

Conceivably, Leu may cause a decrease in the plasma concentration of other amino acids by promoting their utilization in tissues directly (Buse and Weigand, 1977) or through insulin (Fulks of al. 1975). Membrane transport effects have also been proposed (Eriksson et al.. 1981: Nyhan et al., 1961) although they have received little attention. Insulin could also act by stimulating membrane transport (Shotwell et al.. 1983).

Under our experimental conditions, the amino acids studied were likely to be exposed to an insulin effect. according to the time course of glucose and insulin concentration changes. Although the latter were modest (data not shown), it is accepted that insulin variations in plasma are more properly interpreted by measuring the glucose levels simultaneously (Young and Kavam, 1991). Accordingly, the sharp increase in the insulin/glucose ratio and its prevention by diazoxide points to an 
insulin-releasing effect of Leu (Figure 2B). This is consistent with previous work that showed that Leu is a potent insulin secretagogue in the rat (Landgraf et al., 1974). Although a direct effect cannot be ruled out at this time, it is likely that the effects of diazoxide observed here are due to its well known inhibitory effect on insulin release (Wolff et al., 1968).

The selective sensitivity of some of these amino acids to diazoxide is, in fact, consistent with insulin effects in humans. Fukagawa et al. (1986), using the euglycaemic insulin clamp technique, showed that Ile and Pro were the most sensitive among several amino acids. Moreover, in the same work these authors found that Gly and Ala concentrations in plasma were unaffected by insulin, as was observed in the present study. Contrary to our findings, however, they found a statistically significant decrease in Phe and Tyr plasma concentrations after insulin infusion (Fukagawa et al. 1986). We believe that a possible insulin contribution to the decrease in the plasma concentrations of Phe and Tyr in the present study was masked by a stronger effect of Leu in the utilization of these two aromatic amino acids. This interpretation is consistent with the observed enhanced incorporation of $\left[{ }^{14} \mathrm{C}\right] \mathrm{Tyr}$ into protein, promoted by Leu in isolated rat diaphragm (Buse and Weigand, 1977). The decrease of Ala in plasma, clearly insulin-independent in this study, can also be explained by a direct effect of Leu on the utilization of this amino acid in muscle, as could be the case for Met also.

Eriksson et al. (1981) found a small but statistically significant increase and decrease in plasma insulin and glucose concentrations, respectively, after Leu infusion to healthy human adults. Concomitantly these authors found a statistically significant decrease in the plasma levels of all the amino acids studied here, among others. However, they raised the possibility of stimulated exchange diffusion of the intracellular Leu with the plasma large neutral amino acids through transport system $L$ (Eriksson et al., 1981). Since we were particularly interested in net changes in amino acid distribution. our experimental design here does not allow us to exclude such a mechanism of stimulated exchange diffusion, which may be a very early event after Leu administration. In the present study, Gly was the only amino acid that showed a statistically significant increase in its distribution ratio muscle/plasma (Table 1). The parallel finding that only Gly showed a statistically significant decrease and increase. respectively, in its plasma concentration and in its distribution ratio muscle/plasma after aBCH administration (Figure 1) strongly suggests that the corresponding decrease of Gly in plasma caused by Leu is due to trapping of this amino acid by inhibition of its exodus from muscle cells through system $\mathrm{L}$, according to the hypothesis proposed here. This explanation is again consistent with early and recent work in our laboratory showing that Gly, Ala and Pro seem particularly prone to be trapped into muscle and liver cells in the presence of excesses of Phe (de Céspedes er al., 1989, their figs. 3 and 4) and other system L substrates or analogues (Christensen et al., 1948). This effect probably arises at least in part from the readiness of the entry of the small neutral amino acids through $\mathrm{Na}^{+}$-dependent system $\mathrm{A}$, a movement parallel, but in the opposite direction, to the one occurring through system $L$ in muscle and liver (Christensen, 1987; de Céspedes et al., 1989). Accordingly, the statistically significant decrease in Pro plasma concentration may be explained by 
transport effects as shown by the statistically significant increase in its distribution ratio liver/plasma. Since the distribution ratio liver/plasma for Gly also showed a statistically significant increase, its trapping in liver may make an additional contribution to its decrease in plasma. Moreover, this apparent enhancement of transport of Pro and Gly in liver is not observed in the presence of diazoxide. Since transport system $A$ has been shown to be responsive to insulin in isolated preparations of liver and muscle (Shotwell et al., 1983), a role for insulin in stimulating the entry of Pro and Gly through system A in liver seems likely. Although not statistically significant, the increase in the distribution ratio liver/plasma, and its relative decrease in the presence of diazoxide, is also observed for Ala.

We have no explanation at this time for the mechanism of the increased distribution ratios liver/plasma for Ile, Val, Phe, Tyr and Met that otherwise is probably contributing to the depletion of these amino acids in plasma. We do not know either why these increases, with the exception of $\mathrm{Tyr}$, are all unaffected by diazoxide.

A stimulatory effect of the enzymes of the branched-chain amino acid catabolic pathways has been proposed as a mechanism of the Leu-induced decrease in the plasma concentrations of Ile and Val (Phansalker et al., 1970). If this mechanism were to operate in MSUD, some residual stimulative activity of the branched-chain ketoacid decarboxylase should still be present. Insulin levels have been reported as normal in patients with MSUD (Soltesz et al., 1983). Since in the present study, the observed Leu-induced decrease of Ile and Val in plasma and muscle was prevented by diazoxide, insulin seems to play a role in this effect, at least under our experimental conditions. Utilization of Leu itself in liver and muscle, probably for protein synthesis (Tessari et al., 1987), is apparently enhanced by insulin, since much higher levels were observed in those tissues as well as in plasma in the presence of diazoxide.

Distinguishing among the above mechanisms may be important in guiding attempts to correct amino acid imbalances in hereditary or acquired disease states that may lead to short- or long-term deleterious effects, especially on the brain. We have discussed this subject (Christensen, 1987; de Céspedes et al., 1989). A compensation for such effects might be attained by the administration of selected amino acids or amino acid analogues, or of insulin or insulin antagonists. Further work is needed, however, to assess the significance of the depletion of plasma neutral amino acids, some of them (such as Phe and Tyr) precursors of neurotransmitters or themselves neurotransmitters as is Gly, as a cause of impairment of the function or the structure of the brain.

\section{ACKNOWLEDGEMENT}

Grant DK 33281 and DK25548 from the National Institutes of Health, United States Public Health Service. Dr de Céspedes was working at the University of Michigan under a Fulbright Scholarship. 


\section{REFERENCES}

Buse, M. G. and Weigand, D. A. Studies concerning the specificity of the effect of leucine on the turnover of proteins in muscles of controls and diabetic rats. Biochim. Biophys. Acta 475 (1977) 81-89

Christensen, H. N. Role of membrane transport in interorgan amino acid flows: Where do the depleted amino acids go in phenylketonuria? In Kaufman, S. (ed.) Amino Acids in Health and Disease: New Perspectives, vol. 55, Allan R. Liss, New York, 1987, pp. 1-16

Christensen, H. N. and Cullen, A. M. Intensified gradients for endogenous amino acid substrates for transport system $\mathrm{L}$ on injecting a specific competitor of that system. Life Sci. 29 (1981) $749-753$

Christensen, H. N., Streicher, J. A. and Elbinger, R. L. Effects of feeding individual amino acids upon the distribution of other amino acids between cells and extracellular fluid. $J$. Biol. Chem. 172 (1948) 515-524

de Céspedes, C., Thoene, J. G., Lowler, K. and Christensen, H. N. Inhibition of exodus of small neutral amino acids from non-brain tissues in hyperphenylalaninaemic rats. J. Inher. Metab. Dis. 12 (1989) 166-180

DiGeorge, A. M., Rezvani, I., Garibaldi, L. S. and Schwartz, M. Prospective study of Maple Syrup Urine Disease for the first four days of life. New Engl. J. Med. 307 (1982) 1492-1495

Eriksson, S., Hagenfeldt, L. and Wahren, J. A. A comparison of the effects of intravenous infusion of individual branched chain amino acids on blood amino acid levels in man. Clin. Sci. 60 (1981) 95-100

Fajans, S. S., Floyd, J. C., Knopt, R. F. and Conn, J. W. Effects of amino acids and proteins on insulin secretion in man. Recent Progr. Hormone Res. 23 (1967) 617-662

Fukagawa, N. K., Minaker, K. L., Young, V. R. and Rowe, J. W. Insulin dose-dependent reductions in plasma amino acids in man. Am. J. Physiol. 250 (1986) E13-E17

Fulks, R. M., Li, J. B. and Goldberg, A. L. Effects of insulin, glucose and amino acids on protein turnover in rat diaphragm. J. Biol. Chem. 250 (1975) 290-298

Landgraf, R., Landgraf-Leurs, M. M. C. and Horl, R. L-Leucine and L-phenylalanine induced insulin release and the influence of $\mathrm{D}$-glucose. Kinetic studies with the perfused rat pancreas. Diabetologia 10 (1974) 415-420

Lowry, O. H. and Hastings, A. B. Histochemical changes associated with aging. I. Methods and calculations. J. Biol. Chem. 143 (1942) 257-269

Luck, J. M., Morrison, G. and Wilbur, L. F. The effect of insulin on the amino acid content in blood. $J$. Biol. Chem. 77 (1928) 151-156

Nyhan, W. L., Borden, M. and Childs, B. Idiopathic hyperglycinemia. A new disorder of amino acid metabolism. II. The concentration of other amino acids in plasma and their modification by the administration of leucine. Pediatrics 27 (1961) 539-545

Oxender, D. L. and Christensen, H. N. Distinct mediating systems for the transport of neutral amino acids by the Ehrlich cell. J. Biol. Chem. 238 (1963) 3686-3699

Phansalkar, S. V., Norton, P. M., Holt, L. E. and Snyderman, S. E. Amino acid interrelationships: The effect of a load of leucine on the metabolism of isoleucine. Proc. Soc. Exp. Biol. Med. 134 (1970) 262-263

Rogers, Q. R., Spolter, P. D. and Harper, A. E. Effect of leucine-isoleucine antagonism on plasma amino acid pattern in rats. Arch. Biochem. Biophys. 97 (1962) 497-504

Rossi-Fanelli, F., Cangiano, C., Capocaccia, L., Cascino, A., Ceci, F., Muscaritoli, M. and Giunchi, G. Use of branched chain amino acids for treating hepatic encephalopathy: Clinical experiences. Gut 27 (suppl. 1) (1986) 111-115

Shotwell, M. A., Kilberg, M. S. and Oxender, D. L. The regulation of neutral amino acid transport in mammalian cells. Biochim. Biophys. Acta 737 (1983) 267-284

Snyderman, S. E., Norton, P. M., Roltman, E. and Holt, L. E. Maple Syrup Urine Disease with particular reference of dietotherapy. Pediatrics 34 (1964) 454-472

Soltesz, G., Jenkins, P. A. and Aynsley-Green, A. Hypoglycaemia in classical maple syrup urine disease is not due to hyperinsulinism. J. Inher. Metab. Dis. 6 (1983) 178 
Tager, H. S. and Christensen, H. N. Hyperglycemic action of 2-aminonorbornane-2-carboxylic acid in the rat. Biochem. Biophys. Res. Commun. 44 (1971) 185-191

Tager, H. S. and Christensen, H. N. 2-Aminonorbornane-2-carboxylic acid. Preparation, properties and identification of the four isomers. J. Am. Chem. Soc. 94 (1972) 969-972

Tessari, P., Ichiostro, S., Biolo, G., Trevisan, R., Fantin, G., Marescotti, M. C., Iori, E., Tiengo, A. and Crepaldi, G. Differential effects of hyperinsulinemia and hyperaminoacidemia on leucine-carbon metabolism in vivo. Evidence for distinct mechanisms in regulation of net amino acid deposition. J. Clin. Invest. 79 (1987) $1062-1069$

Wilkinson, L. Systat: The System for Statistics, SYSTAT Inc., Evanston, IL, 1986

Winter, C. G. and Christensen, H. N. Migration of amino acids across the membrane of human erythrocyte. J. Biol. Chem. 239 (1964) 872-878

Wolf, F., Hirsh, E., Wales, J. and Viktora, J. Experimental and clinical studies with diazoxide. Ann. N.Y. Acad. Sci. 150 (1968) 429-441

Young, C. W. and Kavam, J. H. Hypoglycemic disorders. In Greenspan, F. S. (ed.) Basic and Clinical Endocrinology, 3rd edn, Appleton and Lange, Norwalk, 1991, pp. 657-658 